\title{
Diversidad y biomasa de la selva circundante al Volcán de los Murciélagos, en Campeche, México
}

\section{Diversity and biomass of the tropical forest surrounding the Volcán de los Murciélagos in Campeche, Mexico}

\author{
Ligia Guadalupe Esparza-Olguín ${ }^{1 *}$, Jorge A. Vargas-Contreras 2 [0, Eduardo Martínez-Romero $\sqrt[3]{ }$, Griselda \\ Escalona-Segura ${ }^{1}$ (i) \\ ${ }^{1}$ Departamento Conservación de la Biodiversidad, El Colegio de la Frontera Sur; Avenida Rancho, Polígono 2-A, Ciudad Industrial \\ Lerma, CP. 24500. Campeche, Campeche, México. \\ ${ }^{2}$ Facultad de Ciencias Químico Biológicas, Universidad Autónoma de Campeche, Avenida Agustín Melgar s/n, Colonia Buenavista, CP. \\ 24030. Campeche, Campeche, México. \\ ${ }^{3}$ Investigación y Soluciones Socioambientales A.C., Calle 4, núm. 4, Colonia Lázaro Cárdenas, CP. 24095. Campeche, Campeche, \\ México. \\ *Autor de correspondencia: lesparza@ecosur.mx, Igeo.ecosur@gmail.com
}

Artículo científico recibido: 06 de septiembre de 2018 aceptado: 24 de octubre de 2018

RESUMEN. La diversidad de la vegetación aledaña al refugio El Volcán de los Murciélagos no ha sido descrita, por lo que el objetivo fue determinar su composición florística, diversidad estructural y la biomasa almacenada. Se establecieron ocho sitios de muestreo, cercanos y lejanos al borde. Se censaron 2844 individuos en un área de muestreo de $5000 \mathrm{~m}^{2}$. La riqueza de especies fue de 84, distribuidas en 34 familias. Las especies más abundantes fueron Croton oerstedianus, Eugenia winzerlingii y Drypetes lateriflora, siendo Astronium graveolens la única especie en la NOM-059-SEMARNAT-2010. El área basal varió entre 9.55 y $26.05 \mathrm{~m}^{2} \mathrm{ha}^{-1}$ y la densidad entre 4288 y 8272 ind ha ${ }^{-1}$. La estructura horizontal y vertical, presentan un patrón en forma de J-invertida. Los valores de diversidad estructural tienen una relación positiva con la acumulación de biomasa, que varió entre 4.23 y $51.95 \mathrm{Mg} \mathrm{ha}^{-1}$. Los resultados indican que la heterogeneidad y diversidad de la vegetación circundante a la cueva El Volcán de los Murciélagos, constituyen características del hábitat que influyen en las especies de murciélagos que habitan la cueva. Las condiciones estructurales y la diversidad correlacionados con la acumulación de biomasa, refuerzan la necesidad de generar estrategias de conservación para este refugio multiespecífico.

Palabras clave: Calakmul, carbono, diversidad estructural, refugio multiespecífico.

ABSTRACT. The diversity of vegetation surrounding the El Volcán de los Murciélagos refuge has not been previously described; therefore, the objective of this study was to determine its floristic composition, structural diversity and stored biomass. Eight sampling sites were established, both near and far from the rim. A total of 2,844 individuals were counted in a $5000 \mathrm{~m}^{2}$ sampling area. Species richness was 84, distributed in 34 families. The most abundant species were Croton oerstedianus, Eugenia winzerlingii and Drypetes lateriflora, with Astronium graveolens being the only species in NOM-059SEMARNAT-2010. The basal area varied between 9.55 and $26.05 \mathrm{~m}^{2} \mathrm{ha}^{-1}$ and the density between 4288 and 8272 ind $\mathrm{ha}^{-1}$. The horizontal and vertical structure have an inverted $\mathrm{J}$-shaped pattern. The structural diversity values have a positive relationship with biomass accumulation, which varied between 4.23 and $51.95 \mathrm{Mg} \mathrm{ha}^{-1}$. Results indicate that the heterogeneity and diversity of the vegetation surrounding the El Volcán de los Murciélagos cave constitute habitat characteristics that influence the bat species that inhabit the cave. The structural conditions and diversity correlated with biomass accumulation reinforce the need to generate conservation strategies for this multi-specific refuge.

Key words: Calakmul, carbon, structural diversity, multi-specific refuge. 


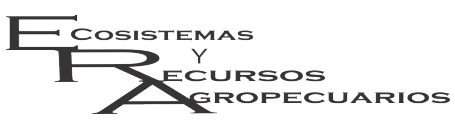

\section{INTRODUCCIÓN}

La cueva El Volcán de los Murciélagos es un refugio multiespecífico de alta abundancia (EscalonaSegura et al. 2002, Torres-Flores y Santos-Moreno 2017). A pesar de estar considerada como un sitio estratégico para la conservación, por albergar nueve especies de murciélagos, una nectívora (Glossophaga soricina) y ocho insectívoras (Pteronotus parnellii, P. personatus, P. davyi, P. gymnonotus, Mormoops megalophylla, Natalus mexicanus, Myotis keaysi y Nyctinomops laticaudatus), con poblaciones de más de tres millones de individuos. Pero aun con su importancia no se han realizado trabajos que documenten las condiciones de conservación de este refugio (Vargas-Contreras et al. 2012). La cueva, al igual que otros sitios en la región de Calakmul, está expuesta a disturbios como visitas no reguladas, construcción o ampliación de carreteras, contaminación por plaguicidas, cambios en la cubierta vegetal, entre otros (Amábilis-Sosa y Benítez 2014). En este sentido, una de las características más importantes en el entorno de la cueva es la condición de la vegetación que hasta ahora no se ha documentado y que juega un papel importante en las especies de quirópteros (Escalona-Segura et al. 2002, García-García y Santos-Moreno 2014). En el caso de los murciélagos, se ha documentado que la estructura y composición de la vegetación influye de manera importante en su movimiento, dado que navegan y forrajean al interior de ésta (Jung et al. 2012). Las condiciones de la vegetación también se asocian con la disponibilidad de alimento, de sitios de percha y la protección contra depredadores (Hayes y Loeb 2007). La conservación de la selva circundante a este refugio multiespecífico de alta abundancia también provee otros servicios como la captura de carbono (Balvanera 2012).

La vegetación circundante a la cueva se ha descrito como selva mediana subperennifolia con vegetación secundaria (Escalona-Segura et al. 2002), de la cual no se han realizado estudios que describan la vegetación y su condición, por lo que el objetivo del presente trabajo fue analizar la composición florística, la diversidad estructural y la biomasa (carbono) almacenado en esta vegetación, para conocer las características de este refugio y generar estrategias de conservación.

\section{MATERIALES Y MÉTODOS}

\section{Área de estudio}

La cueva El Volcán de los Murciélagos se ubica en las coordenadas $18^{\circ} 31^{\prime} 22.15^{\prime \prime}$ LN y $89^{\circ} 49^{\prime} 25.07$ " LO, en una loma cárstica cercana al kilómetro 107.5 de la Carretera Federal 186 Escárcega-Chetumal. El clima es cálido subhúmedo con lluvias en verano $\left(A w_{1}\right)$, temperatura media anual de $24.4{ }^{\circ} \mathrm{C}$ y precipitación de $1141 \mathrm{~mm}$. Los suelos corresponden a leptosoles y vertisoles pélicos de texturas finas (García y Secaira 2006). La vegetación circundante a la cueva es selva mediana subperennifolia y vegetación secundaria derivada de ésta (EscalonaSegura et al. 2002).

\section{Muestreo y obtención de datos en campo}

Para el muestreo de la vegetación circundante a la cueva, se trazaron ocho sitios de muestreo de 25 $x 25 \mathrm{~m}\left(625 \mathrm{~m}^{2}\right)$ para un total de $5000 \mathrm{~m}^{2}$ muestreados. La distribución de los sitios de muestreo se realizó en relación con los puntos cardinales, teniendo al borde de la cueva cuatro sitios (borde cercano) y los restantes a una distancia entre $325 \mathrm{~m}$ y $500 \mathrm{~m}$ (borde lejano). Los cuadros ubicados al sur $(325 \mathrm{~m}) \mathrm{y}$ al oeste $(400 \mathrm{~m})$, no pudieron ubicarse a $500 \mathrm{~m}$ de distancia del borde de la cueva debido a la presencia de la Carretera Federal 186 EscárcegaChetumal. Cada cuadro se subdividió en cuadros de $5 \times 5 \mathrm{~m}$ para facilitar el censo de los individuos leñosos perennes (árboles y arbustos) con diámetro normal (Dn) mayor de $1 \mathrm{~cm}$ a una altura de $1.3 \mathrm{~m}$. Para cada individuo se registró el nombre científico, común, Dn y la altura total.

\section{Análisis de los datos}

Se realizó un listado florístico de las especies leñosas para conocer la composición de especies en cada sitio de muestreo y condición (borde cercano y lejano), para corroborar y en su caso actualizar los datos taxonómicos se usó la base de datos 


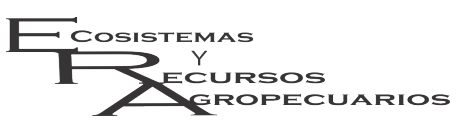

The Plant List (2013). También se estimaron los parámetros estructurales básicos: i) densidad de individuos, definida como el número de individuos de cada especie en una hectárea y ii) área basal calculada como: $B A=\sum \pi\left(D n_{i} / 2\right)^{2}$. Mientras que la estructura horizontal, evaluada con el Dn y vertical mediante la altura total se analizó con las distribuciones de frecuencia agrupadas por clase, empleando la fórmula $K=1+3.33 \times \log (n)$. Donde: $\mathrm{K}$ es el número de clases y $n$ el número de árboles.

Para estimar la diversidad en cada sitio de muestreo y condición (borde cercano y lejano) se estimó la riqueza de especies y se calculó la diversidad con el índice de diversidad de Shannon-Wiener, que combina el número de especies y la igualdad de la distribución de los individuos entre todas las especies, por lo que expresa la uniformidad entre las especies de la muestra (Magurran 2004, Begon et al. 2006). A partir de los valores de equitatividad (J') se comparó que tan bien se ajusta la diversidad observada con la diversidad máxima esperada. Los índices se calcularon con las siguientes fórmulas (Magurran 2004):

$$
H^{\prime}=-\sum P i * \log P i
$$

Donde: $\mathrm{H}=$ Índice de Shannon-Wiener, $\mathrm{Pi}=$ Abundancia relativa, y Log $=$ Logaritmo base 10

$$
J^{\prime}=\frac{H^{\prime}}{H_{M A X}}
$$

Donde: $\mathrm{H}_{M A X}=\log \mathrm{S}$ y $\mathrm{H}^{\prime}=$ Índice de ShannonWiener

La diversidad estructural se estimó con el índice de Shannon-Wiener para las especies (Hs), el índice de Shannon-Wiener por clases diamétricas (Hd), el índice de Shannon-Wiener por clases de altura $(\mathrm{Hh})$ y el índice promedio de la diversidad estructural (Hsdh). Los cuales se calcularon con las siguientes fórmulas (Lei et al. 2009, MartínezSánchez 2016):

$$
H s=\sum_{i=1}^{m} \times \log p i
$$

Donde: $p i$ = proporción del área basal para la especie i, y $\mathrm{m}$ = número de especies
Esparza-Olguín et al.
Diversidad y Biomasa de la selva

Ecosist. Recur. Agropec. 6(16):79-90,2019

$$
H d=\sum_{i=1}^{d} \times \log p i
$$

Donde: $\mathrm{pi}=$ proporción del área basal por clase la diamétrica i, y $\mathrm{d}=$ número de clases diamétricas

$$
H h=\sum_{i=1}^{h} \times \log p i
$$

Donde: $\mathrm{pi}=$ proporción del área basal para la clase de altura $\mathrm{i}, \mathrm{y} \mathrm{h}=$ número de clases de altura

Para determinar diferencias significativas entre los valores de diversidad del Índice de ShannonWinner y los índices de diversidad estructural, se realizaron verificaciones por pares entre los ocho sitios de muestreo, aplicando una prueba de $t$-student modificada (Hutcheson 1970, Zar 2010).

Para la estimación de la biomasa se emplearon ecuaciones alométricas generadas para áreas con condiciones similares (tipo cobertura vegetal), eligiendo ecuaciones empleadas por Aryal et al. (2014), Esparza-Olguín y Martínez-Romero (2018). La fórmula propuesta por Cairs et al. (2013), modificada por Urquiza-Haas et al. (2007), se usó para estimar la biomasa (AGB) de individuos con diámetros normales $(\mathrm{Dn})$ mayores o iguales a $10 \mathrm{~cm}$ :

$$
A G B=\frac{\left(\exp \left(-2.12605+0.868 \ln \left(D^{2} H\right)\right) *\left(\frac{\rho_{1}}{\rho_{m}}\right)\right)}{10^{3}}
$$

Donde: $\mathrm{D}=$ diámetro del tronco a $1.30 \mathrm{~m}$ de altura, $\mathrm{H}=$ altura total del árbol, $\rho_{1}=$ densidad de madera por árbol $\left(\mathrm{g} / \mathrm{cm}^{3}\right)$, y $\rho_{m}=$ Promedio de densidad de madera de árboles usado para generar la ecuación $(0.75 \mathrm{~g} / \mathrm{cm} 3)$

En el caso de los individuos con Dn entre 5 y $9.9 \mathrm{~cm}$ se usó la siguiente fórmula (Chave et al. 2005) para la estimación de biomasa:

$$
A G B=\frac{\left(\exp \left(-2.1875+0.916 \ln \left(\rho D^{2} H\right)\right)\right)}{10^{3}}
$$

Donde: $\mathrm{D}$ es el diámetro del tronco a $1.30 \mathrm{~m}$ de altura, $\mathrm{H}$ es la altura total del árbol y $\rho$ la densidad de madera por árbol $\left(\mathrm{g} / \mathrm{cm}^{3}\right)$.

Para los individuos de menos de $5 \mathrm{~cm}$ de diámetro normal se utilizó la fórmula propuesta por Hughes et al. (1999): 


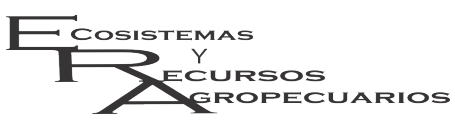

$$
A G B=\frac{\left(\exp \left(4.9375+1.0583 \ln \left(D^{2}\right)\right) \times 1.14\right)}{10^{6}}
$$

Donde: $\mathrm{D}$ corresponde al diámetro del tronco a la altura de $1.30 \mathrm{~m}$

El valor de densidad de madera por especie arbórea se asignó en función de los datos reportados para estas especies por Chave et al. (2006) y Zanne et al. (2009). En el caso de las especies que no tienen reportado el dato de densidad de madera, se usó el valor promedio de densidad de madera por sitio de muestreo empleando la siguiente fórmula:

$$
\sum \frac{\sum\left(B A_{i} * W S G_{i}\right)}{\sum B A}
$$

Donde: $\mathrm{BAi}=$ Área basal por individuo, $\mathrm{WSGi}=$ Densidad de madera por especie con valores conocidos $\left(\mathrm{g} / \mathrm{cm}^{3}\right)$.

La estimación de biomasa por sitio de muestreo se calculó con la sumatoria de la biomasa de cada uno de los individuos presentes. La cantidad de carbono contenido en el componente aéreo de cada individuo se calculó con la conversión de biomasa a carbono con el factor 0.47 (Fonseca et al. 2011).

\section{RESULTADOS}

\section{Composición vegetal}

Se registraron 2844 individuos de 84 especies y 34 familias en un área de muestreo de $5000 \mathrm{~m}^{2}$ circundantes a la cueva El Volcán de los Murciélagos. Las familias con mayor riqueza de especies fueron Fabaceae (16 especies), Rubiaceae (8), Euphorbiaceae (7) y Sapindaceae (4), representado en conjunto el $41.66 \%$ de las especies reportadas. De las 84 especies encontradas, el $10.71 \%$ se presentaron en los ocho sitios de muestreo; mientras que el $18.82 \%$ fueron especies únicas, que se presentaron solo en uno de los sitios. Las especies que destacaron por su abundancia fueron Croton oerstedianus (300 individuos), Eugenia winzerlingii (209), Drypetes lateriflora (199), Malpighia lundelli (166) y Acacia angustissima (111). La única es-
Esparza-Olguín et al. Diversidad y Biomasa de la selva Ecosist. Recur. Agropec. 6(16):79-90,2019

pecie en la NOM-059-SEMARNAT-2010 fue Astronium graveolens.

\section{Estructura vegetal}

La densidad de individuos varió entre 4288 y 8272 ind ha $^{-1}$, mientras el área basal $(\mathrm{AB})$ osciló entre 9.55 y $26.05 \mathrm{~m}^{2}$ ha $^{-1}$ (Tabla 1), en ambos casos con valores significativamente mayores para los sitios de muestreo de borde lejano $(Z=2.48, p=$ 0.013 y $Z=2.52, p=0.012$, respectivamente). Las estructuras horizontales (categorías diamétricas) de los sitios de muestreo cercanos y lejanos al borde presentaron en todos los casos un patrón en forma de J-invertida, donde la mayor proporción de individuos se encuentra en las categorías con Dn menor o igual a $5 \mathrm{~cm}$. Para los sitios cercanos al borde entre el 57 y el $77 \%$ de los individuos tienen un Dn menor o igual a $5 \mathrm{~cm}$, en tanto que en los sitios lejanos al borde ocupan entre el 66 y el $77 \%$ (Figura 1). En tanto que las categorías con individuos con diámetros mayores a $21 \mathrm{~cm}$ representan entre el 1 y el $4 \%$, tanto en sitios de borde lejano como de borde cercano, con especies como Brosimum alicastrum, A. graveolens y A. tomentosa.

La estructura vertical (categorías de altura) de la vegetación en los sitios cercanos y lejanos al borde presentó un patrón en que la proporción de individuos fue inversamente proporcional a la altura, lo que indica que en todos los casos la mayor proporción de individuos, entre 79 y $95 \%$ se encuentran en las categorías de alturas menores o iguales a 8 $\mathrm{m}$ (Figura 2). Por otro lado, las categorías mayores a $14 \mathrm{~m}$ representan entre 1 y $4 \%$, destacando especies como A. graveolens, Thouinia paucidentata, B. alicastrum y $A$. tomentosa.

\section{Diversidad florística y estructural}

La riqueza de especies en los sitios cercanos al borde varió entre 28 y 37 especies, la cual fue significativamente menor que la presente en los sitios lejanos al borde, donde los valores fueron entre $44 \mathrm{y}$ 53 especies ( $F=24.12, p=0.0027)$. Los valores del índice de diversidad de Shannon-Wiener oscilaron entre 2.42 y 3.15 en los sitios cercanos al borde de la cueva y entre 3.06 y 3.29 en los sitios lejanos a la 
Tabla 1. Parámetros estructurales y de diversidad en los cuadros estudiados aledaños a la cueva El Volcán de los Murciélagos, Campeche.

\begin{tabular}{|c|c|c|c|c|c|c|c|c|c|}
\hline Sitio & $\mathrm{D}\left(\right.$ ind ha $\left.{ }^{-1}\right)$ & $\mathrm{AB}\left(\mathrm{m}^{2} \mathrm{ha}^{-1}\right)$ & S & $\mathrm{H}_{\log 10}^{\prime}$ & J' & $\mathrm{Hs}$ & $\mathrm{Hd}$ & $\mathrm{Hh}$ & Hsdh \\
\hline C1 & 4624 & 11.58 & 35 & 3.023 & 0.850 & 1.18 & 0.86 & 0.72 & 0.92 \\
\hline C2 & 4288 & 19.21 & 37 & 3.154 & 0.873 & 0.92 & 0.78 & 0.66 & 0.79 \\
\hline C3 & 4560 & 9.55 & 37 & 2.417 & 0.669 & 1.08 & 0.81 & 0.62 & 0.84 \\
\hline C4 & 4768 & 14.65 & 28 & 2.647 & 0.794 & 0.96 & 0.80 & 0.57 & 0.78 \\
\hline C5 & 8272 & 26.05 & 47 & 3.136 & 0.815 & 1.19 & 0.83 & 0.75 & 0.92 \\
\hline C6 & 5296 & 17.98 & 44 & 3.056 & 0.808 & 1.21 & 0.85 & 0.76 & 0.94 \\
\hline $\mathrm{C} 7$ & 5456 & 23.41 & 49 & 3.292 & 0.846 & 1.26 & 0.83 & 0.69 & 0.93 \\
\hline $\mathrm{C} 8$ & 8240 & 16.35 & 53 & 3.256 & 0.820 & 1.27 & 0.87 & 0.75 & 0.96 \\
\hline
\end{tabular}

Los sitios C1 (N), C2 (S), C3 (O), C4 (E) corresponden a los sitios cercanos al borde y los C5 (N), C6 (S), C7 (O), C8 (E) a los sitios lejanos al borde. D: densidad de individuos; AB: área basal; S: riqueza de especies; $\mathrm{H}_{\log 10}$ : índice de Shannon-Wiener; J': índice de equitatividad; Hs: índice de Shannon-Wiener para las especies; Hd: índice de Shannon-Wiener por clases diamétricas; Hh: índice de Shannon-Wiener por clases de altura; Hsdh: índice promedio de la diversidad estructural.

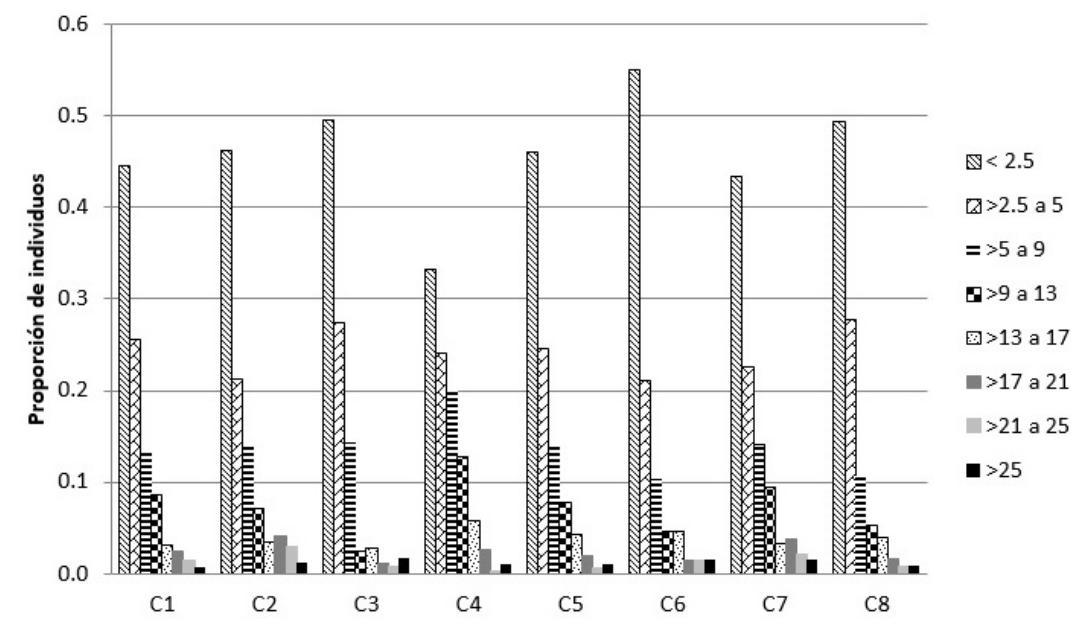

Figura 1. Estructura horizontal en los sitios analizados al borde de la cueva "El Volcán de los Murciélagos". Los sitios C1 (N), C2 (S), C3 (O), C4 (E) corresponden a los sitios cercanos al borde y los C5 (N), C6 (S), C7 (O), C8 (E) a los sitios lejanos al borde.

cueva (Tabla 1), sin diferencias significativas $(\mathrm{t}=1.83$, $\mathrm{p}=0.109$ ). La equitatividad varió entre 0.67 y 0.87 en los sitios cercanos al borde y de 0.81 a 0.84 en los sitios lejanos al borde (Tabla 1).

Los valores de diversidad estructural estimados mediante el índice de Shannon-Wiener para las especies (Hs), variaron entre 0.91 y 1.27 , en general los sitios del borde cercano presentaron valores significativamente menores que los del borde lejano (Tabla 1). El mismo patrón se presentó con los valores del índice de diversidad estructural por clases diamétricas $(\mathrm{Hh})$ que osciló entre 0.57 y 0.76 ; sin diferencias significativas entre los valores del índice de ShannonWiener por categorías de altura Hd (Tabla 1). El índice promedio de la diversidad estructural (Hsdh) tuvo valores entre 0.78 y 0.96 .

\section{Biomasa y carbono acumulado}

La biomasa acumulada en la vegetación aledaña a la cueva El Volcán de los Murciélagos varió entre 4.23 y $51.95 \mathrm{ha}^{-1}$, y el carbono acumulado entre 1.99 y $24.42 \mathrm{Mg} \mathrm{ha}^{-1}$; siendo en ambos casos significativamente menores los valores en los sitios del borde cercano (Tabla 2). En relación con la biomasa y el carbono acumulados en las diferentes clases diamétricas, se observó que las categorías con hasta $9 \mathrm{~cm}$ de Dn contienen menos del 10\%, salvo en el caso de los sitios de borde cercano (5 y 7$)$ con más del $15 \%$. Por otro lado, las categorías con más de $17 \mathrm{~cm}$ almacenan más del $40 \%$ de la biomasa 


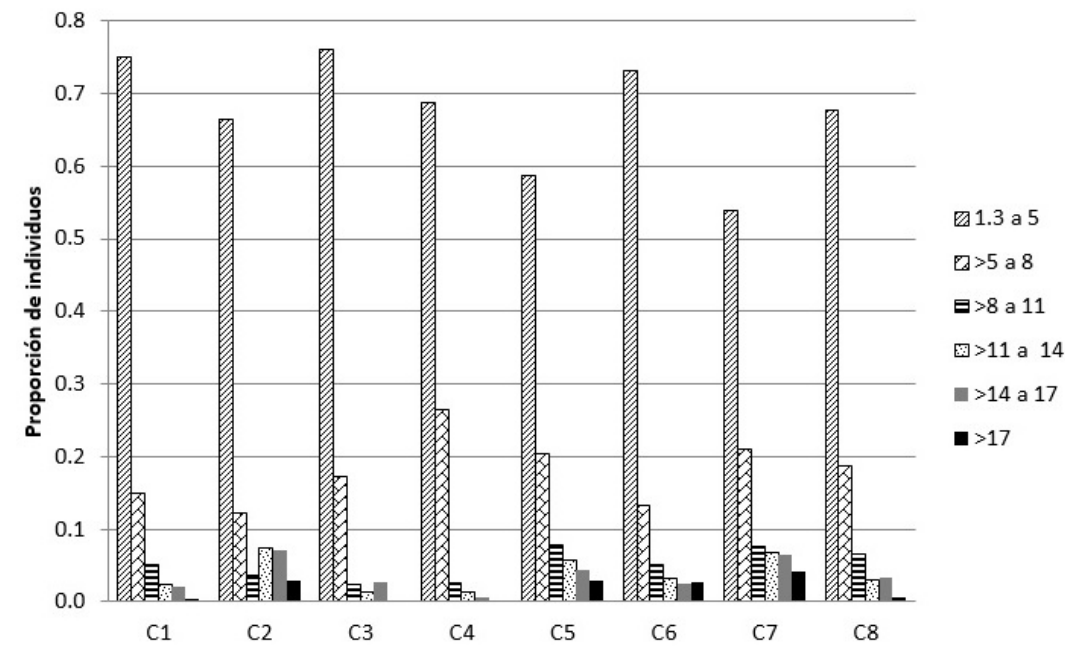

Figura 2. Estructura vertical en los sitos al borde de la cueva "El Volcán de los Murciélagos". Los sitios C1 (N), C2 (S), C3 (O), C4 (E) corresponden a los sitios cercanos al borde y los C5 (N), C6 (S), C7 (O), C8 (E) a los sitios lejanos al borde.

Tabla 2. Biomasa y carbono acumulados por sitio de muestreo y categoría diamétrica.

\begin{tabular}{|c|c|c|c|c|c|c|c|c|}
\hline & C1 & C2 & C3 & C4 & C5 & C6 & $\mathrm{C} 7$ & C8 \\
\hline Biomasa (t ha-1) & 15.48 & 14.05 & 5.53 & 4.23 & 32.80 & 32.13 & 26.97 & 51.95 \\
\hline Carbono $\left(\mathrm{MgC} \mathrm{ha}^{-1}\right)$ & 7.27 & 6.60 & 2.60 & 1.99 & 15.40 & 15.10 & 12.68 & 24.42 \\
\hline \multicolumn{9}{|c|}{ Categorías diamétricas (biomasa / carbono) } \\
\hline \multirow[t]{2}{*}{$<2.5$} & 0.063 & 0.056 & 0.061 & 0.051 & 0.125 & 0.091 & 0.072 & 0.131 \\
\hline & 0.030 & 0.026 & 0.029 & 0.024 & 0.059 & 0.043 & 0.034 & 0.062 \\
\hline \multirow[t]{2}{*}{$>2.5$ a 5} & 0.183 & 0.136 & 0.203 & 0.175 & 0.312 & 0.176 & 0.212 & 0.333 \\
\hline & 0.086 & 0.064 & 0.095 & 0.082 & 0.147 & 0.083 & 0.100 & 0.157 \\
\hline \multirow[t]{2}{*}{$>5$ a 9} & 0.563 & 0.809 & 0.602 & 0.742 & 1.616 & 0.638 & 1.147 & 1.116 \\
\hline & 0.265 & 0.380 & 0.283 & 0.349 & 0.760 & 0.300 & 0.539 & 0.525 \\
\hline \multirow[t]{2}{*}{$>9$ a 13} & 1.295 & 1.341 & 0.115 & 2.057 & 5.345 & 1.362 & 11.095 & 2.633 \\
\hline & 0.608 & 0.630 & 0.054 & 0.967 & 2.512 & 0.640 & 5.214 & 1.237 \\
\hline \multirow[t]{2}{*}{$>13$ a 17} & 3.454 & 1.542 & 1.047 & 0.317 & 12.826 & 2.332 & 2.663 & 26.943 \\
\hline & 1.623 & 0.725 & 0.492 & 0.149 & 6.028 & 1.096 & 1.252 & 12.663 \\
\hline \multirow[t]{2}{*}{$>17$ a 21} & 3.730 & 3.065 & 1.672 & 0.570 & 0.976 & 18.380 & 5.303 & 10.200 \\
\hline & 1.753 & 1.441 & 0.786 & 0.268 & 0.459 & 8.639 & 2.493 & 4.794 \\
\hline \multirow[t]{2}{*}{$>21$ a 25} & 0.546 & 5.826 & 0.067 & 0.010 & 1.749 & 0.545 & 5.885 & 4.338 \\
\hline & 0.257 & 2.738 & 0.032 & 0.005 & 0.822 & 0.256 & 2.766 & 2.039 \\
\hline \multirow[t]{2}{*}{$>25$} & 5.642 & 1.276 & 1.759 & 0.310 & 9.850 & 8.605 & 0.592 & 6.252 \\
\hline & 2.652 & 0.600 & 0.826 & 0.146 & 4.630 & 4.044 & 0.278 & 2.938 \\
\hline
\end{tabular}

Los sitios C1 (N), C2 (S), C3 (O), C4 (E) corresponden a los sitios cercanos al borde y los C5 (N), C6

(S), C7 (O), C8 (E) a los sitios lejanos al borde.

y el carbono total.

\section{Diversidad estructural vs Biomasa acumulada}

La correlación entre la diversidad estructural (Hs, Hd y Hh) y la acumulación de biomasa (AGB) en la vegetación leñosa aledaña a la cueva es significativa y positiva, es decir, a mayor diversidad estructural mayor cantidad de biomasa acumulada (Figuras 3 , 4 y 5). La correlación más fuerte se presenta con el índice de Shannon-Wiener por clases de altura (Figura 4), seguida por la diversidad estructural medida como el índice de Shannon-Wiener por especies (Figura 3).

\section{DISCUSIÓN}

\section{Composición vegetal}

La composición florística leñosa en el área de 


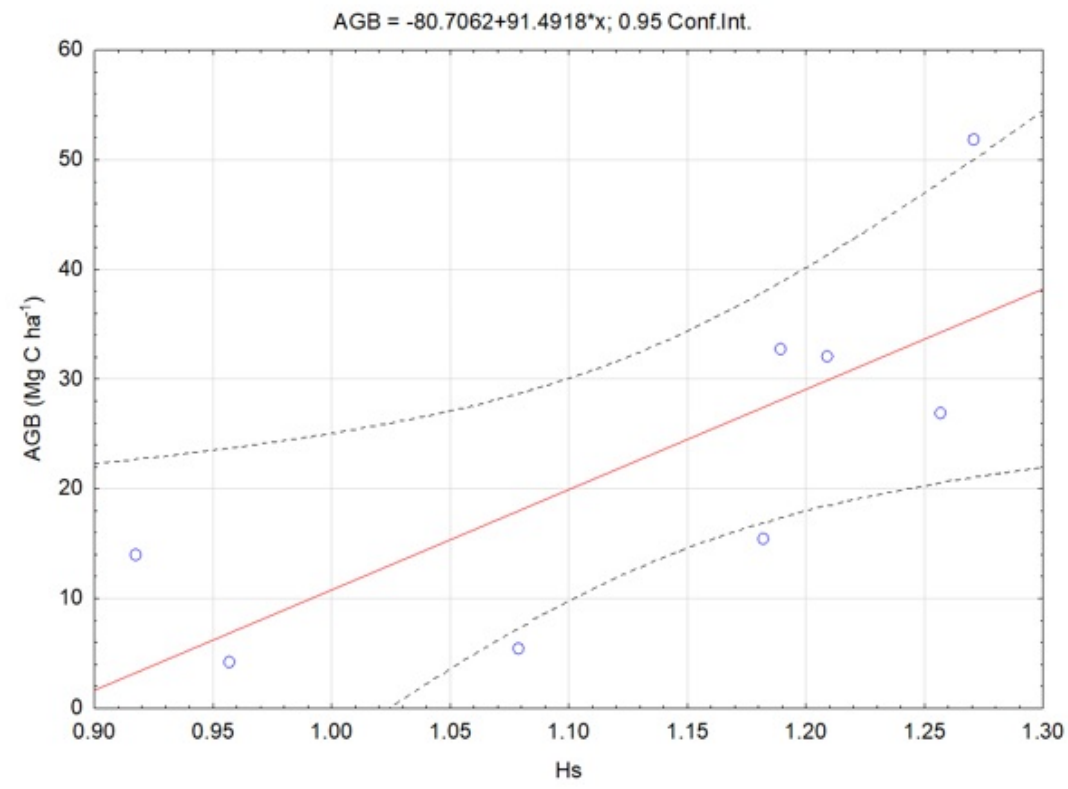

Figura 3. Relación entre la biomasa acumulada y la diversidad estructural medida con el índice de Shannon para especies en la vegetación aledaña a la cueva El Volcán de los Murciélagos.

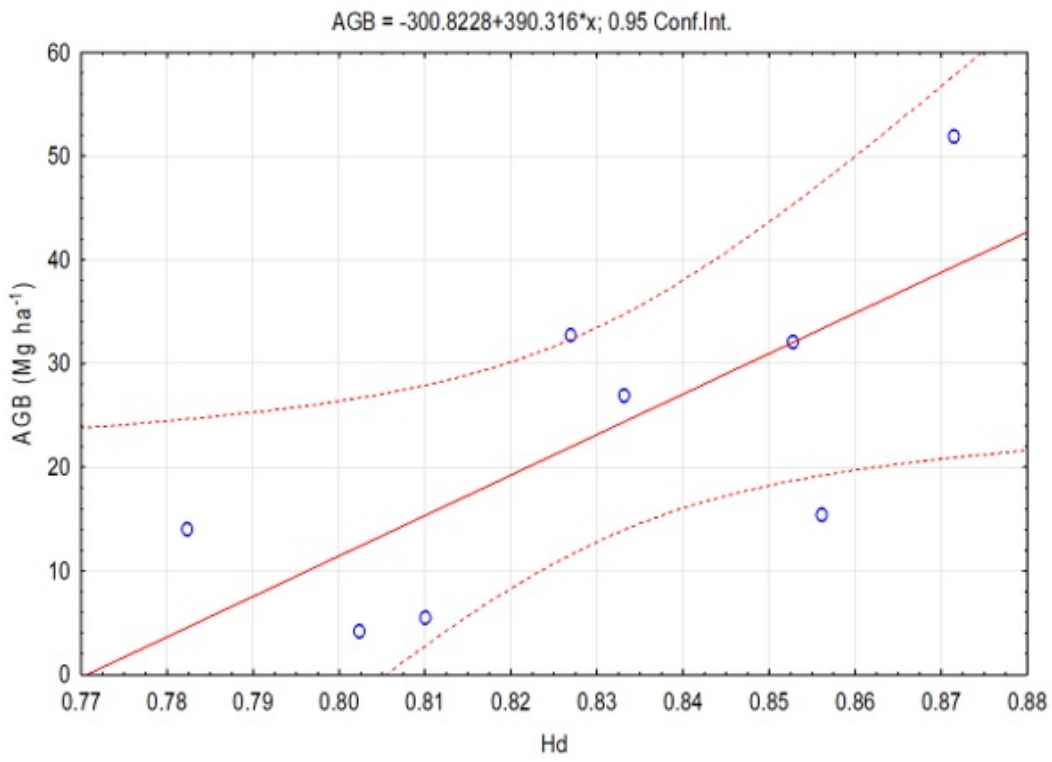

Figura 4. Relación entre la biomasa acumulada y la diversidad estructural medida con e índice de Shannon para categorías diamétricas en la vegetación aledaña a la cueva El Volcán de los Murciélagos.

estudio representa el $19.4 \%$ de las especies y el $50.7 \%$ de las familias de árboles reportadas para la península de Yucatán por Ibarra-Manríquez et al. (1995). Estos resultados son semejantes a los re- portados por Díaz-Gallegos et al. (2002), quienes señalan para una selva baja subperennifolia en la Región de Calakmul la presencia de 1660 individuos y 65 especies, es decir el $77.4 \%$ de la riqueza re- 


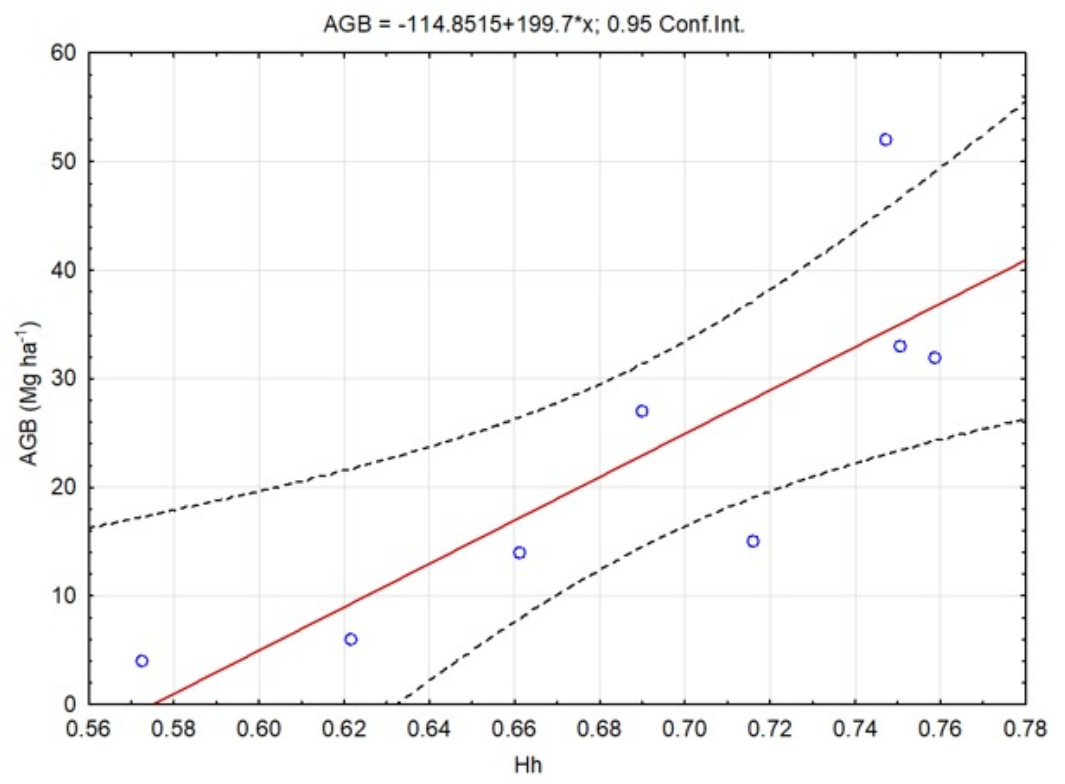

Figura 5. Relación entre la biomasa acumulada y la diversidad estructural medida con el índice de Shannon para categorías de altura en la vegetación aledaña a la cueva El Volcán de los Murciélagos.

portada para este estudio con un $42.6 \%$ menos de individuos. Un patrón semejante fue reportado por García-Licona et al. (2014), que mencionan para selvas en el ejido El Carmen II en Calakmul, 7.2\% menos especies y $35.14 \%$ menos individuos que lo encontrado en este estudio. Por otro lado, ZamoraCrescencio et al. (2012) registraron para Oxpemul, 91 especies (9.2\%) más que lo reportado en este estudio. Mientras que Esparza-Olguín y Martínez-Romero (2018) reportan para selvas del ejido Álvaro Obregón en Calakmul una mayor riqueza de especies (90) con densidad de individuos semejante (5 170 ind ha ${ }^{-1}$ ). Las diferencias en el número de individuos y familias pueden asociarse al tamaño del área muestreada, que fue distinto en todos los estudios, a las condiciones microambientales y de relieve, así como a las historias de uso y de disturbios.

Las familias con mayor número de especies en este estudio (Fabaceae, Rubiaceae, Euphorbiaceae y Sapindaceae) se encuentran entre las diez especies más frecuentes y con mayor número de especies reportadas para la península de Yucatán por Ibarra-Manríquez et al. (1995) y Carnevali et al. (2010); entre los estudios reportadas para la selva de la península de Yucatán, Sánchez-Sánchez et al. (2007) reportan la composición y estructuras vegetal en una selvas del norte de Quintana Roo, mientras que Zamora-Crescencio et al. (2012), García-Licona et al. (2014) y Esparza-Olguín y Martínez-Romero (2018) describen la vegetación de selvas en la región de Calakmul.

\section{Estructura vegetal}

Para la estructura vertical y horizontal de la vegetación, la distribución en forma de $\mathrm{J}$ invertida es un elemento que permite señalar que la vegetación circundante a la cueva El Volcán de los Murciélagos, presenta procesos de regeneración adecuados, que garantizan el reemplazo de árboles que pudieron ser eliminados por diversas causas. Estos resultados coinciden con lo reportado para selvas subperennifolias de la región de Calakmul en diferentes condiciones sucesionales (Zamora-Crescencio et al. 2012, Gutiérrez-Báez et al. 2011, García-Licona et al. 2014, Esparza-Olguín y Martínez-Romero 2018). Considerando que la abundancia en las clases diamétricas mayores $(>20 \mathrm{~cm})$ se incrementa conforme avanza el proceso sucesional, es decir con- 


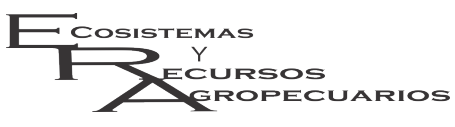

forme la vegetación se recupera de un disturbio, se puede inferir que los sitios lejanos al borde presentan mejores condiciones en la vegetación. Estos resultados coinciden con los reportados por Ferguson et al. (2003) para selvas de El Petén guatemalteco, GarcíaLicona et al. (2014), Esparza-Olguín y MartínezRomero (2018) para selvas de la región de Calakmul y van Breugel et al. (2006) para selvas de Marqués de Comillas, Chiapas. La heterogeneidad en la estructura horizontal y vertical podría contribuir a la gran cantidad de murciélagos que se albergan en la cueva, al considerar que se ha reportado que la abundancia y diversidad de murciélagos se relaciona de forma positiva con la heterogeneidad y diversidad florística en bosques, además de influir en su conducta particularmente en lo relacionado con el vuelo (Aranguren et al. 2011, Jung et al. 2012). Los valores de área basal reportados en este estudio son en general menores a los reportados por otros autores para selvas de la región de Calakmul (García-Licona et al. 2014, Esparza-Olguín y Martínez-Romero 2018) quienes reportan valores entre 22.6 y $37.4 \mathrm{~m}^{2} \mathrm{ha}^{-1}$.

Los datos de la estructura concuerdan con diferentes autores que señalan la existencia de manchones de vegetación que se encuentran en fases de recuperación luego de haber sido afectados por la agricultura, tala e incendios, lo que significa cambio en la estructura y composición vegetal de las selvas medianas subperennifolias (Vester et al. 2007, García-Licona et al. 2014, EsparzaOlguín y Martínez-Romero 2018). En el caso de la vegetación circundante a la cueva El Volcán de los Murciélagos, los disturbios más importantes han sido la construcción de la carretera Escárcega - Chetumal, y la presencia de grupos de turistas que la visitan.

\section{Diversidad florística y estructural}

La diversidad en los sitios analizados fue semejante a la reportada por otros autores para las selvas subperennifolias de la región de Calakmul (Díaz et al. 2002, Zamora-Crescencio et al. 2012) y mayor que la encontrada por García-Licona et al. (2014) y Esparza-Olguín y Martínez-Romero (2018). La diversidad estructural analizada con los índices de Shannon-Wiener, muestra que los sitios lejanos al borde son estructuralmente más diversos en relación con la composición de especies, estructura por categorías diamétricas y de altura que los sitios cercanos al borde; lo que permite inferir que existe un impacto diferencial de disturbios como las visitas no reguladas y la construcción y ampliación de la carretera en la vegetación del borde lejano y cercano a la cueva. Los valores de diversidad estructural estimados fueron menores a los reportados por Martínez-Sánchez et al. (2015) y MartínezSánchez (2016) para selvas medianas subperenifolias en áreas protegidas de Tabasco, y semejantes a los reportados por Esparza-Olguín y MartínezRomero (2018) para selvas en la región de Calakmul en las cuales se ha realizado manejo forestal. La alta diversidad presente en la vegetación aledaña a la cueva refuerza la necesidad de que este sitio se encuentre bajo vigilancia para garantizar la conservación de la biodiversidad que alberga.

\section{Biomasa y carbono acumulado}

La biomasa y el carbono acumulado en la vegetación leñosa circundante a la cueva es semejante a lo reportado por otros autores para vegetación secundaria derivada de selva mediana subperennifolia en la región de Calakmul con valores que oscilan entre $24.94 \mathrm{Mg} \mathrm{ha}^{-1}\left(11.72 \mathrm{Mg} \mathrm{C}^{-1}\right.$ ) y $61.53 \mathrm{Mg}$ ha $^{-1}$ (28.92 Mg C ha-1) (Lawrence y Foster 2003, Eaton y Lawrence 2008, Aryal et al. 2014, EsparzaOlguín y Martínez-Romero 2018). Para los sitios en el borde cercano los valores corresponden a los reportados para vegetación secundaria en fases tempranas de la sucesión, es decir entre 5 y 10 años de recuperación (Aryal et al. 2014). En tanto que los valores estimados para los sitios en el borde lejano son semejantes a los estimados para vegetación secundaria en fase intermedia (10 a 15 años) de la sucesión (Lawrence y Foster 2003, Eaton y Lawrence 2008, Aryal et al. 2014, Esparza-Olguín y MartínezRomero 2018). Estos resultados pueden reflejar cambios ocurridos en la vegetación como resultado de distintos disturbios a los que está expuesta la vegetación aledaña a la cueva como visitas no reguladas, construcción o ampliación de la carretera y el acueducto. 


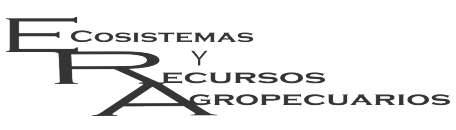

Esparza-Olguín et al. Diversidad y Biomasa de la selva Ecosist. Recur. Agropec. 6(16):79-90,2019

\section{Diversidad estructural vs Biomasa acumulada}

La relación positiva entre la diversidad estructural y la acumulación de biomasa encontrada en el área estudiada es congruente con lo reportado por Esparza-Olguín y Martínez-Romero (2018) para selvas subperennifolias de la región de Calakmul y por Martínez-Sánchez et al. (2015) y Martínez-Sánchez (2016) para selvas de Tabasco. Estos resultados permiten señalar que hay una relación positiva entre la biodiversidad y la productiva primaria, medida como acumulación de biomasa, en la vegetación aledaña a la cueva; lo que refuerza la importancia de conservar este sitio.

\section{CONCLUSIONES}

La vegetación circundante a la cueva El Volcán de los Murciélagos es secundaria derivada de selva subperennifolia, probablemente resultado de disturbios como visitas sin regulación y la construcción de la carretera y el acueducto. La heterogeneidad y diversidad de la vegetación constituyen características del hábitat que necesitan las distintas especies de murciélagos que habitan la cueva. Estas características pueden jugar un papel importante en la diversidad de murciélagos presente y con su conducta, en lo referente al vuelo. La acumulación de biomasa, carbono, diversidad y heterogeneidad en la vegetación aledaña a la cueva, refuerzan la necesidad de generar estrategias de conservación para este refugio multiespecífico de alta abundancia.

\section{AGRADECIMIENTOS}

A los parataxónomos Demetrio Álvarez y Manuel Arana por el apoyo para la identificación de especies. A la Secretaría de Medio Ambiente y Aprovechamiento Sustentable del Gobierno del Estado de Campeche por facilitar el ingreso y las actividades de investigación en la cueva El Volcán de los Murciélagos. El proyecto fue financiado por FomixCampeche proyecto 125406.

\section{LITERATURA CITADA}

Amábilis-Sosa LE, Benítez JA (2014) Efecto de borde; ruido, materia orgánica en suelo y contaminantes persistentes en las áreas naturales protegidas de Calakmul-Balamkú, Campeche. Revista AIDIS de Ingeniería y Ciencias Ambientales: Investigación, desarrollo y práctica 7: 201-209.

Aranguren Cl, González-Carcacía JA, Martínez H, Nassar JM (2011) Noctilio albiventris (Noctilionidae), a potential seed disperser in disturbed tropical dry forest habitats. Acta Chiropterologica 13: 189-194.

Aryal DR, de Jong BHJ, Ochoa-Gaona S, Esparza-Olguín L, Mendoza-Vega J (2014) Carbon stocks and changes in tropical secondary forests of southern Mexico. Agriculture, Ecosystems and Environment 195: 220-230.

Balvanera P (2012) Los servicios ecosistémicos que ofrecen los bosques tropicales. Ecosistemas 21: 136-147.

Begon M, Townsend CR, Harper JL (2006) Ecology from individuals to ecosystems. 4th edition. Blackwell Publishing Ltd. Malden, MA, USA. 759p.

Cairns MA, Olmsted I, Granados J, Argaez J (2003) Composition and aboveground tree biomass of a dry semievergreen forest on Mexico's Yucatan Peninsula. Forest Ecology and Management 186: 125-132.

Carnevali FC, Tapia-Muñoz GJL, Duno de Stefano R, Ramírez Morillo I (2010) Flora llustrada de la Península de Yucatán: Listado Florístico. 1ra Edición. Centro de Investigación Científica de Yucatán. Mérida, Yucatán, México. 328p.

Chave J, Andalo C, Brown S, Cairns MA, Chambers JQ, Eamus D, et al. (2005) Tree allometry and improved estimation of carbon stocks and balance in tropical forest. Oecologia 145: 87-99.

Chave J, Muller-Landau HC, Baker TR, Easdale TA, ter Steege H, Webb CO (2006) Regional and phylogenetic variation of wood density across 2456 Neotropical tree species. Ecological Applications 16: 2356-2367. 
Díaz-Gallegos JR, Castillo-Acosta O, García-Gil G (2002) Distribución espacial y estructura arbórea de la selva baja subperennifolia en un ejido de la Reserva de la Biosfera de Calakmul, Campeche, México. Universidad y Ciencia 18: 11-28.

Eaton JM, Lawrence D (2008) Loss of carbon sequestration potential after several decades of shifting cultivation in the Southern Yucatán. Forest Ecology and Management 258: 949-958.

Escalona-Segura G, Vargas-Contreras JA, Interián-Sosa L (2002) Registros importantes de mamíferos para Campeche, México. Revista Mexicana de Mastozoología 6: 166-170.

Esparza-Olguín L, Martínez-Romero E (2018) Diversidad y carbono almacenado en el área forestal permanente de Álvaro Obregón, Campeche. Revista Mexicana de Ciencias Forestales 9: 152-186.

Ferguson BG, Vandermeer J, Morales H y Griffith DM (2003) Post-Agricultural succession in El Petén, Guatemala. Conservation Biology 17: 818-828.

Fonseca W, Rey JM, Alice FE (2011) Carbon accumulation in the biomass and soil of different aged secondary forests in the humid tropics of Costa Rica. Forest Ecology and Management 262: 1400-1408.

García-García JL, Santos-Moreno A (2014) Efectos de la estructura del paisaje y de la vegetación en la diversidad de murciélagos filostómidos (Chiroptera: Phyllostomidae) de Oaxaca, México. Revista Biología Tropical 62: 217-239.

García G, Secaira F (2006) Una visión para el futuro: cartografía de las selvas Maya, Zoque y Olmeca. Primera Edición. Infoterra Editores. San José, Costa Rica. 40p.

García-Licona JB, Esparza-Olguín L, Martínez-Romero E (2014) Estructura y composición de la vegetación leñosa de selvas en diferentes estadios sucesionales en el ejido El Carmen II, Calakmul, México. Polibotánica 38: 1-26.

Gutiérrez-Báez C, Ortíz-Díaz JJ, Flores-Guido JS, Zamora-Crescencio P (2011) Diversidad, estructura y composición de las especies leñosas de la selva mediana subcaducifolia del punto de unión territorial (Put) de Yucatán, México. Polibotánica 33: 151-174.

Hayes JP, Loeb SC (2007) The influence of forest management on bats in North America. In: Lackie MJ, Hayes JP, Kurta A (ed) Bats in forests: Conservation and management. John Hopkins University Press, Baltimore, MD. pp: 207-236.

Hughes RF, Kauffman JB, Jaramillo VJ (1999) Biomass, carbon, and nutrient dynamics of secondary forests in humid tropical region of México. Ecology 80: 1892-1907.

Hutcheson K (1970) A test for comparing diversities based on the Shannon formula. Journal of Theoretical Biology 29: 151-154.

Ibarra-Manríquez G, Villaseñor JL, Durán-García R (1995) Riqueza de especies y endemismos del componente arbóreo de la Península de Yucatán, México. Boletín de la Sociedad Botánica de México 57: 49-77.

Jung K, Kaiser S, Böhm S, Nieschulze J, Kalko E (2012) Moving in three dimensions: effects of structural complexity on occurrence and activity of insectivorous bats in managed forest stands. Journal of Applied Ecology 49: 523-531.

Lawrence D, Foster DR, (2003) Recovery of nutrient cycling and ecosystem properties following swidden cultivation: regional and stand-level constraints. In: Turner II BL, Geoghegan J, Foster DR (ed). Integrated Land-Change Science and Tropical Deforestation in Southern Yucatán. Final Frontiers, Oxford University Press. New York, USA. pp: 81-104. 
Lei X, Wang W, Peng C (2009) Relations hips between stand growth and structural diversity in spruce-dominated forest in New Brunswick, Canada. Canadian Journal of Forest Research 39: 1835-1847.

Magurran AE (2004) Measuring biological diversity. First Edition. Blackwell Science Ltd. Oxford, UK. 264p.

Martínez-Sánchez JL, Tigar BJ, Cámara L, Castillo O (2015) Relationship between Structural Diversity and Carbon Stocks in Humid and SubHumid Tropical Forest of Mexico. Ecoscience 22: 125-131.

Martínez-Sánchez JL (2016) Comparación de la diversidad estructural de una selva alta perennifolia y una mediana subperennifolia en Tabasco, México. Madera y Bosques 22: 29-40.

Sánchez-Sánchez O, Islebe GA, Valdez-Hernández M (2007) Flora arbórea y caracterización de gremios ecológicos en distintos estados sucesionales de la selva mediana de Quintana Roo. Foresta Veracruzana 9: 17-26.

Torres-Flores JW, Santos-Moreno A (2017) Inventory, features, and protection of underground roots used by bats in Mexico. Acta Chiropterologica 19: 439-454.

The Plant List (2013). Version 1.1. http://www.theplantlist.org/ . Fecha de consulta 7 de julio de 2018.

Urquiza-Haas T, Dolman PM, Peres CA (2007) Regional scale variation in forest structure and biomass in the Yucatan Peninsula, Mexico: Effects of forest disturbance. Forest Ecology and Management 247: 80-90.

van Breugel M, Martínez-Ramos M, Bongers F (2006) Community dynamics during early secondary succession in Mexican tropical rain forest. Journal of Tropical Ecology 22: 663-674.

Vargas-Contreras JA, Escalona-Segura G, Arroyo-Cabrales J, Rendon von Osten J, Navarrol L (2012) Conservación de Murciélagos en Campeche. Therya 3: 53-66.

Vester HFM, Lawrence D, Eastman R, Turner II BL, Calme S, Dickson R et al. (2007) Land change in the Southern Yucatán and Calakmul Biosphere Reserve: effects on habitat and biodiversity. Ecological Applications 17: 989-1003.

Zamora-Crescencio P, Gutiérrez-Báez C, Folan WJ, Domínguez-Carrasco MR, Villegas P, Cabrera-Mis G, et al. (2012) La vegetación leñosa del sitio arqueológico de Oxpemul, municipio de Calakmul, Campeche, México. Polibotánica 33: 131-150.

Zanne AE, López-González G, Coomes DA, llic J, Jansen S, Lewis SL, et al. (2009) Global Wood Density Database. Dryad Identififier. htpp:/hdl.handle.net/1025/dryad.235. Fecha de consulta 12 de julio de 2018.

Zar JH (2010) Biostatistical analysis. 5th Edition. Prentice-Hall. New Jersey, USA. 944p. 